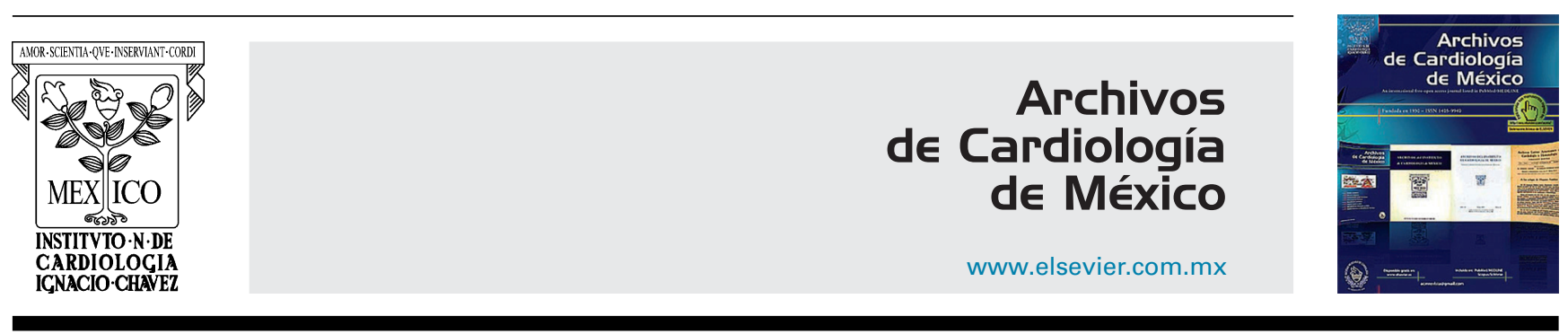

ARTÍCULO DE REVISIÓN

\title{
Hipertensión portopulmonar: Revisión actualizada
}

\author{
Nielzer Rodríguez-Almendros ${ }^{\mathrm{a}}$, Liz N. Toapanta-Yanchapaxi ${ }^{\mathrm{b}}$, \\ Jonathan Aguirre Valadez ${ }^{\mathrm{b}}$, Nilda Espinola Zavaleta ${ }^{\mathrm{c}}$, Sergio G. Muñoz-Martínez ${ }^{\mathrm{b}}$ \\ e Ignacio García-Juárez ${ }^{\mathrm{b}, *}$
}

\author{
a Departamento de Hipertensión Pulmonar y Función Ventricular Derecha, UMAE Cardiología, Centro Médico Nacional Siglo XXI, \\ Instituto Mexicano del Seguro Social, México, D.F., México \\ b Departamento de Gastroenterología, Instituto Nacional de Ciencias Médicas y Nutrición Salvador Zubirán, Ciudad de México, \\ México \\ c Departamento de Ecocardiografia, Instituto Nacional de Cardiología Ignacio Chávez, Ciudad de México, México
}

Recibido el 6 de junio de 2016; aceptado el 8 de noviembre de 2016

\section{PALABRAS CLAVE}

Hipertensión arterial pulmonar;

Cirrosis hepática;

Trasplante de hígado; México

\section{KEYWORDS}

Pulmonary arterial hypertension; Liver cirrhosis; Liver transplantation; Mexico

\begin{abstract}
Resumen La hipertensión portopulmonar (HPP) es una entidad poco frecuente a nivel mundial, aunque se desconocen los datos epidemiológicos en México. Sin embargo, las enfermedades crónicas del hígado son muy prevalentes en mexicanos. La HPP es el $4 .^{\circ}$ subtipo en frecuencia del grupo de la hipertensión arterial pulmonar. Su diagnóstico está dentro de 2 escenarios: los pacientes con sospecha de hipertensión pulmonar y los candidatos a trasplante hepático ortotópico (THO). Tanto el ecocardiograma como el cateterismo cardiaco derecho son determinantes para el diagnóstico en ambos escenarios. La HPP es un reto para el THO, pues aumenta la mortalidad perioperatoria de manera importante. El uso de terapia específica es la piedra angular de este padecimiento, como una medida para poder mejorar el desenlace de los que llegan a ser candidatos a un THO con HPP moderada a grave. Es importante reconocer que la HPP puede llegar a ser una contraindicación para el THO. Hasta el momento el papel del trasplante combinado pulmón-hígado o corazón-pulmón-hígado como una medida de curación de la enfermedad vascular pulmonar en pacientes con HPP es incierto.

( 2016 Instituto Nacional de Cardiología Ignacio Chávez. Publicado por Masson Doyma México S.A. Este es un artículo Open Access bajo la licencia CC BY-NC-ND (http://creativecommons. org/licenses/by-nc-nd/4.0/).
\end{abstract}

\section{Portopulmonary hypertension: Updated review}

Abstract Portopulmonary hypertension (PPH) is a rare condition worldwide, although epidemiological data are unknown in Mexico. However, chronic liver diseases are very prevalent in Mexico. PPH is the 4th subtype in frequency in the group of pulmonary arterial hypertension. Its diagnosis is made within 2 scenarios: patients with suspected pulmonary hypertension and candidates

* Autor para correspondencia. Vasco de Quiroga 15, Sección XVI, Del. Tlalpan. C.P. 14000. Teléfono.: +5215522481329.

Correo electrónico: drinter77@gmail.com (I. García-Juárez). 
for orthotopic liver transplantation (OLT). Both echocardiogram and a right cardiac catheterisation are crucial for diagnosis in both cases. PPH is a challenge for OLT, since it can significantly increase perioperative mortality. The use of specific therapy is the cornerstone of this disease, as a measure to improve the outcome of those who become candidates for OLT with moderate to severe PPH. It is important to recognise that PPH can be a contraindication to OLT. The role of lung-liver transplantation or heart-lung-liver transplantation as a measure to heal pulmonary vascular disease in patients with PPH is still uncertain.

(C) 2016 Instituto Nacional de Cardiología Ignacio Chávez. Published by Masson Doyma México S.A. This is an open access article under the CC BY-NC-ND license (http://creativecommons. org/licenses/by-nc-nd/4.0/).

\section{Introducción}

El hígado en condiciones estables recibe el $15-20 \%$ del gasto cardiaco, de los cuales 2 terceras partes provienen del sistema venoso portal (circuito de baja presión) ${ }^{1}$. La hipertensión portal (HPo) es común y se caracteriza por un aumento en el gradiente de presión entre la vena porta y la cava inferior ${ }^{2}$, lo que hemodinámicamente se define por un gradiente de presión venosa hepática (GPVH) $\geq 6 \mathrm{~mm} \mathrm{Hg}$, una presión transesplénica $\geq 15 \mathrm{~mm} \mathrm{Hg}$ y/o una presión de la vena porta $\geq 21 \mathrm{~mm} \mathrm{Hg}^{1}$. Su etiología puede variar a lo largo del tiempo, la edad y estado socioeconómico de la población de un país ${ }^{3}$, sin embargo, la causa más frecuente es la cirrosis hepática $(\mathrm{CH})$ o HPo hepática ${ }^{2}$. Se estima que en Occidente corresponde al $88-90 \%$ de los casos, mientras que en el Lejano Oriente al $70 \%^{4-6}$.

\section{La enfermedad hepática crónica y la ascendencia}

Hasta el momento lo que conocemos en México de la hipertensión portopulmonar (HPP) es solo información proveniente de países desarrollados, cuyo control en enfermedades crónicas del hígado son eficientes.

En 2010, el 2\% del total de muertes en el mundo estuvo relacionada con la $\mathrm{CH}$, lo que representa un aumento bruto del $0.4 \%$ desde 1980 , pero una disminución del $21.6 \%$ de la tasa estandarizada por edad en estos últimos 30 años. México no está exento de esta disminución (28.3\%), sin embargo, la tasa de mortalidad (38.3/100,000 habitantes en 2010) es la más alta de América Latina y la octava a nivel mundial (Egipto es el número uno con 72.7/100,000 habitantes) ${ }^{7}$.

A pesar del mestizaje, resultado de la Conquista de América, en España las enfermedades crónicas del hígado se mantuvieron como la causa número 11 de mortalidad en el 2011, con cerca del $1.2 \%$ del porcentaje total de defunciones $^{8}$. Independientemente del crecimiento de la población hispana en los Estados Unidos de América (EUA) (54 millones en 2013 con un 64\% de ascendencia mexicana) ${ }^{9}$, la $\mathrm{CH}$ y las enfermedades crónicas del hígado no estuvieron dentro de las 10 primeras causas de muerte en 2010 en la población general, y al realizar el análisis por grupos étnicos de ascendencia, se encontraron cambios en la mortalidad por dichas enfermedades; por ejemplo, el total de muertes en afroamericanos fue del $0.9 \%$, de blancos no hispanos fue del $1.2 \%$, en nativos americanos del $5.1 \%$ y en hispanos del $3 \%{ }^{10}$. Existe información que hace diferente el área conocida como la zona fronteriza mexicoestadounidense $(100 \mathrm{~km}$ de ancho a cada lado de la frontera), de tal forma que en el lado estadounidense por lo menos el $50 \%$ de la población es hispana, y de estos mayormente descendientes de mexicanos, en esta población las enfermedades crónicas del hígado en 2007 fueron la novena causa de mortalidad (10.618.9/100, 000 habitantes), mientras en el lado mexicano fueron la séptima causa (17.6-29.8/100,000 habitantes) ${ }^{11}$.

En México de acuerdo al Instituto Nacional de Estadística y Geografía, la $\mathrm{CH}$ y otras enfermedades crónicas del hígado representan la quinta causa de muerte en la población general desde el 2000 hasta el 2013 (un 6.3\% del total en 2000, un $5.5 \%$ en 2010 y un $5.5 \%$ en 2013). De acuerdo a la distribución por género, en hombres representa la $5 .^{a}$ causa, y en mujeres entre la $7 .^{\mathrm{a}}$ y $6 .^{\mathrm{a}}$ causa, de acuerdo al año analizado. Por grupos de edad, de 35 a 44 años pasó de

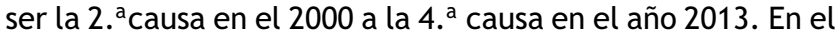
grupo de 45 a 64 años representa la $4 .{ }^{a}$ causa de mortalidad, en este mismo grupo es la $3 .^{a}$ causa en hombres y la $4 .^{a}$ en mujeres. Si se realiza un análisis combinado, en el grupo de 35 a 44 años, vemos que para el 2000 , el $6.5 \%$ de las muertes en mujeres fue por enfermedades crónicas del hígado, para el 2010 fue del $4.9 \%$ y para el 2013 fue del 4.7\%. Mientras que, en hombres dentro del mismo grupo etario, representó el $18.8,12.4$ y $13.8 \%$ para los años 2000, 2010 y 2013 respectivamente. Una tendencia similar se aprecia en el grupo de 45 a 64 años, donde en mujeres para el 2000 la mortalidad fue del $6.9 \%$, en 2010 fue del $6 \%$ y en 2013 fue del $6.1 \%$, mientras que en hombres se aprecian cifras del $17.2 \%$ (2000), 14\% (2010) y 14\% (2013). De acuerdo a estos datos, la $\mathrm{CH}$ es un verdadero problema de salud en México $^{12}$.

\section{Conceptos en relación con hipertensión portopulmonar}

Es importante diferenciar la hipertensión pulmonar (HP), la hipertensión arterial pulmonar (HAP) y la HPP. Así, la HP es un aumento de la presión media de la arteria pulmonar (PMAP), mientras que la HAP es un subtipo de HP y corresponde al grupo 1 de la Organización Mundial de la Salud (OMS), mientras la HPP es parte del grupo de HAP (tabla 1). 
Tabla 1 Definición hemodinámica de hipertensión pulmonar

\begin{tabular}{|c|c|c|}
\hline Definición & Características $^{a}$ & Grupo clínico OMS \\
\hline HP & $\mathrm{PMAP} \geq 25 \mathrm{~mm} \mathrm{Hg}$ & Todos \\
\hline HP precapilar & $\begin{array}{l}\mathrm{PMAP} \geq 25 \mathrm{~mm} \mathrm{Hg} \\
\mathrm{POAP} / \mathrm{PCAP} \leq 15 \mathrm{~mm} \\
\mathrm{Hg}\end{array}$ & $1,3,4$ y 5 \\
\hline HP poscapilar & $\begin{array}{l}\mathrm{PMAP} \geq 25 \mathrm{~mm} \mathrm{Hg} \\
\mathrm{POAP} / \mathrm{PCAP} \geq 15 \mathrm{~mm} \\
\mathrm{Hg}\end{array}$ & 2 y 5 \\
\hline Aislada & $\mathrm{GDP}<7 \mathrm{~mm} \mathrm{Hg}$ & \\
\hline Combinada con precapilar & $\mathrm{GDP} \geq 7 \mathrm{~mm} \mathrm{Hg}$ & \\
\hline
\end{tabular}

No está de más recalcar que la definición de HP es hemodinámica (tabla 2), y para identificar a los pacientes con HAP se deben de descartar otras causas de HP apoyándose en datos clínicos y auxiliares diagnósticos que sigan un flujograma bien establecido ${ }^{13}$. La importancia de identificar a los pacientes con HAP radica en que es una entidad que condiciona un deterioro progresivo tanto de la circulación pulmonar (remodelación arterial), como del corazón (falla ventricular derecha) ${ }^{14}$.

Los pacientes con $\mathrm{CH}$ se pueden presentar con HP relacionada con el estado hiperdinámico característico, con sobrecarga de volumen y con eventos tromboembólico venosos recurrentes ${ }^{15}$. Sin embargo, la asociación de HPo clínica o hemodinámica e HAP con o sin afección hepática crónica se conoce como HPP ${ }^{13}$, y no está relacionada con la etiología de la enfermedad hepática, ni con la gravedad de la HPo (tabla 3$)^{16}$. Los primeros en describir esta asociación fueron

Tabla 2 Definición y clasificación de la hipertensión portopulmonar

\begin{tabular}{|c|c|}
\hline Definición & Características \\
\hline Hipertensión portopulmonar & $\begin{array}{l}P M A P \geq 25 \mathrm{~mm} \mathrm{Hg} \\
P O A P / P C A P \leq 15 \mathrm{~mm} \mathrm{Hg} \\
R V P>3 U W \\
\left(>240 \mathrm{dyn} / \mathrm{s} / \mathrm{cm}^{-5}\right) \\
0 \\
P M A P \geq 25 \mathrm{~mm} \mathrm{Hg} \\
P O A P / P C A P>15 \mathrm{~mm} \mathrm{Hg} \\
\text { GTP }>12 \mathrm{~mm} \mathrm{Hg}^{\mathrm{a}}\end{array}$ \\
\hline Clasificación & PMAP \\
\hline $\begin{array}{l}\text { Leve } \\
\text { Moderada } \\
\text { Grave }\end{array}$ & $\begin{array}{l}\text { PMAP } \geq 25-34 \mathrm{~mm} \mathrm{Hg} \\
\text { PMAP } 35-44 \mathrm{~mm} \mathrm{Hg} \\
\text { PMAP } \geq 45 \mathrm{~mm} \mathrm{Hg}\end{array}$ \\
\hline
\end{tabular}

GTP: gradiente transpulmonar (=PMAP - POAP/PCAP); PMAP: presión media de la arteria pulmonar; POAP/PCAP: presión de oclusión de la arteria pulmonar/presión en cuña de la arteria pulmonar; RVP: resistencia vascular pulmonar.

Adaptada de Krowka y Rodriguez-Roisin ${ }^{27}$.

a Este grupo de pacientes siempre tiene RVP $>3 \mathrm{UW}$.
Mantz y Craig en $1951^{15}$, y hasta antes de 1998 estaba comprendida en el grupo de etiologías «secundarias» de la HP, mientras que ahora está incluida en el grupo 1 de la OMS, mejor conocido como HAP (tabla 1$)^{13}$.

\section{Comportamiento epidemiológico}

Del grupo 1 de la OMS para HP, los 4 subtipos más frecuentes son la HAP idiopática (HAPI) con el $39-61 \%$ de los casos, las formas de HAP asociada a enfermedades del tejido conectivo (11-28\%), a cardiopatías congénitas (10-20\%) y a HPo (5-10\%). La edad de diagnóstico de la HAPI en la mayoría de los registros es en la $6 .^{\text {a }}$ década de la vida ${ }^{17-19}$, mientras que los casos de HPP se diagnostican alrededor de los 50 años $^{4,20}$, aunque aquellos con etiología no hepática de la HPo son de menor edad $( \pm 40 \text { años de edad })^{8}$. En el registro REVEAL, la relación entre mujeres y hombres en HPP es $1: 1^{20}$, aunque en los casos secundarios a enfermedad hepática autoinmune el predominio es en el género femenino ${ }^{21}$.

En 17,901 autopsias en pacientes con un año de edad o más, el $6.9 \%$ tuvieron $\mathrm{CH}$, y en el $0.73 \%$ de aquellos con $\mathrm{CH}$ se detectaron cambios histopatológicos compatibles con $\mathrm{HAP}^{22}$. Dentro del contexto de la HPP las etiologías más comunes de la $\mathrm{CH}$ son por alcohol ${ }^{4}$, virus de hepatitis $\mathrm{C}$ y hepatitis autoinmune ${ }^{21}$, pero a pesar de que la HPP con HPo no hepática es infrecuente, la trombosis portal ${ }^{4}$ y la HPo idiopática ${ }^{6}$ son las causas más importantes.

En los pacientes con HPo no importa la gravedad de la misma, pues a mayor tiempo de evolución (4-7 años en promedio) la probabilidad de desarrollar HPP se incrementa ${ }^{15,21,23}$. En el caso de los pacientes con una derivación portosistémica quirúrgica, el $65 \%$ padecían $\mathrm{HPP}^{24}$ sin embargo, el tiempo entre el diagnóstico de la HPo y la HPP era mayor que en los casos no operados ${ }^{25}$. En pacientes hospitalizados con HPo y $\mathrm{CH}$, la HPP se detectó en el $2-16 \%$ de los $\operatorname{casos}^{25,26}$.

Con base en ligeras diferencias en cuanto a los criterios diagnósticos hemodinámicos, se reportan prevalencias de HPP del 5.3-8.5\% en los candidatos a trasplante hepático ortotópico $(\mathrm{THO})^{27,28}$. Lo relevante en este grupo es que el $65 \%$ de los casos con una presión sistólica de la arteria pulmonar $(P S A P)>50 \mathrm{~mm} \mathrm{Hg}$ en el ecocardiograma 
Tabla 3 Clasificación actualizada de la hipertensión pulmonar

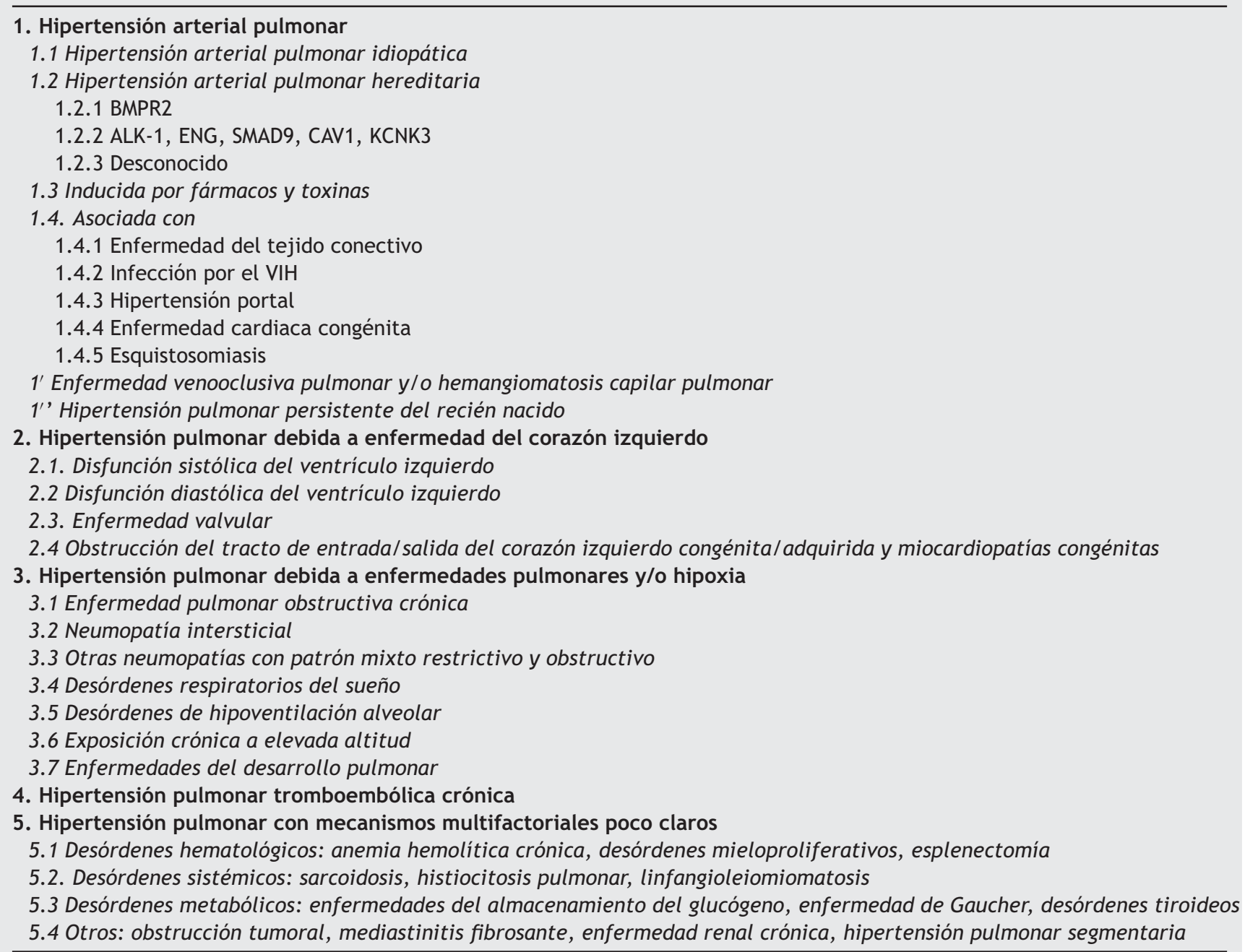

Adaptada de Galiè et al. ${ }^{13}$.

transtorácico (ECOTT) tienen HPP corroborada hemodinámicamente ${ }^{29}$.

\section{Un panorama obscuro de la historia natural}

La supervivencia (SV) media de los pacientes con HPP es de 15 meses. Sin embargo, existen diferencias dependiendo del tipo de HPo subyacente, pues aquellos pacientes del grupo de causa no hepática e HPP tienen una SV $>90 \%$ a 5 años, mientras que en los casos con HPo hepática es del $68 \%$ a 5 años. Esto permite comprender que la mortalidad de los pacientes con HPP depende, principalmente, de la gravedad de la $\mathrm{CH}$ (mayor en Child-Pugh $\mathrm{B} / \mathrm{C}$ ), la hipertensión auricular derecha, un índice cardiaco bajo, una baja saturación venosa central y no recibir tratamiento como epoprostenol ${ }^{4,16}$. Así, las 2 principales causas de muerte en los pacientes con HPP son la descompensación de la falla cardiaca derecha en el $35 \%$ de los casos y las complicaciones relacionadas con la enfermedad hepática en otro $33 \%{ }^{4}$.

La SV a 5 años de los pacientes con HPP es peor que en sus contrapartes con HAPI/HAP hereditaria (HAPH) (40 vs. $64 \%$ ), a pesar de que los valores hemodinámicos sean mejores. Además, el número de hospitalizaciones en la HPP es mayor, y con más riesgo de fallecer después del primer internamiento (78 vs. $70 \% ; p=0.004)^{20}$.

\section{Fisiopatología}

Los hallazgos histopatológicos son indistinguibles de otros subtipos de HAP, en los cuales las arterias pulmonares $<500$ micras son afectadas con un espectro de lesiones obstructivas por todo el pulmón ${ }^{5,30}$.

Al igual que en los otros subtipos de HAP, no hay una explicación fisiopatológica satisfactoria, en parte debido a la falta de un modelo animal y a su baja prevalencia ${ }^{31}$, pero es claro que existe un estímulo detonante y una condición que lo perpetúa. Dentro de los detonantes se han postulado el estado hiperdinámico, el desequilibrio entre sustancias vasoconstrictoras, vasodilatadores y con efecto proliferativo celular en la circulación sanguínea (endotelina 1, interleucina 1 , serotonina, glucagón, tromboxano B2, péptido vasoactivo intestinal e interleucina 6), y factores genéticos ${ }^{32,33}$. Incluso se ha planteado que en pacientes con $\mathrm{CH}$ la condición inflamatoria (endotoxemia, translocación 
bacteriana) puede participar en la remodelación vascular pulmonar $^{34}$.

Las concentraciones de serotonina y endotelina 1 están aumentadas en los pacientes con $\mathrm{CH}$ e HPP (aumento en la síntesis esplácnica). Además, hay un predominio en la circulación pulmonar de receptores tipo A de endotelina 1 (tipo A: vasoconstricción; $\mathrm{B}$ : vasodilatación/degradación de endotelina 1). Otras alteraciones humorales son la disminución en la síntesis de prostaciclina (disminución de la sintetasa en arterias de pequeño y mediano calibre $)^{35}$, y el aumento de la concentración sérica de interleucina 6 (remodelación vascular) con respecto a la interleucina 1 y el factor de necrosis tumoral tipo alfa ${ }^{36}$.

No se ha demostrado que exista relación con mutaciones de la proteína morfogenética ósea tipo 2 (BMPR2), ni de la cinasa similar al receptor de la activina (ALK-1) en los pacientes con $\mathrm{HPP}^{24}$, aunque existen polimorfismos de un solo nucleótido asociados al receptor tipo 1 de estrógeno, a la aromatasa ${ }^{31}$, a la fosfodiesterasa 5 , la angiopoyetina 1 y la proteína de unión al calcio tipo 4. Se ha documentado además mutaciones en las vías del crecimiento celular/apoptosis (proteína S100A4, SERPINE1, RARB, CAV1, SMAD3, RUNX, RBPSUH) ${ }^{16,37}$.

\section{El proceso diagnóstico}

Tempranamente, hasta el $60 \%$ de los casos son asintomáticos $^{38}$ o solo tienen síntomas y signos propios de la HPo. Pese a esto, la mayoría de los pacientes son diagnosticados en clase funcional (CF) III-IV de la clasificación de la New York Heart Association ${ }^{4,17}$ y presentan manifestaciones características de la fase avanzada de la enfermedad, como disnea progresiva de esfuerzo (síntoma predominante de presentación), dolor torácico y/o síncope $^{39}$. También pueden presentar signos de sobrecarga hídrica (ingurgitación yugular, hepatomegalia pulsátil, ascitis, edema de miembros pélvicos) ${ }^{39}$, que en el contexto de la HPo son difíciles de interpretar ${ }^{15}$. Es interesante que los pacientes con HPP no presentan habitualmente acropaquia, asterixis o edema de miembros pélvicos, además se asocian con una menor prevalencia de ascitis con respecto a aquellos con HPo sin HPP ${ }^{21}$.

En el registro REVEAL de los EUA, los pacientes con HPP tuvieron significativamente más fatiga (31 vs. 23\%), edema (33 vs. $21 \%$ ) y distensión abdominal (12 vs. $3 \%$ ) que los pacientes con HAPI/HAPH. La frecuencia de presíncope/síncope fue la misma ${ }^{20}$.

En la exploración física del precordio los posibles hallazgos son: desplazamiento del choque de la punta hacia la línea axilar media, levantamiento sistólico paraesternal izquierdo bajo, palpación del cierre de la válvula pulmonar en el segundo espacio intercostal izquierdo (38\%), auscultación de un desdoblamiento fijo del segundo ruido en el foco pulmonar (82\%), además de un aumento en su intensidad de manera comparativa con el segundo ruido en el foco aórtico, un soplo sistólico en el foco tricuspídeo (61\%) y un soplo diastólico en el foco pulmonar ${ }^{39}$.

También existe el grupo de pacientes con HPP evaluados para THO, los cuales pueden estar asintomáticos, y la única manera de sospechar la enfermedad es mediante los hallazgos del ECOTT, en especial el aumento en la presión sistólica del ventrículo derecho (PSVD) ${ }^{16}$.

Los parámetros de laboratorio no permiten distinguir plenamente a los pacientes con HPP de aquellos con HPo sin HP, aunque los niveles de transaminasas y de fosfatasa alcalina son menores, y la concentración de albúmina es mayor en los casos con HPP ${ }^{21}$. La concentración del fragmento N-terminal del propéptido natriurético cerebral y del péptido natriurético cerebral han mostrado su utilidad en los pacientes con $\mathrm{HAPI}^{40,41}$. En el registro REVEAL no se encontró diferencia en la concentración de ambos entre los pacientes con $\mathrm{HAPI} / \mathrm{HAPH}$ e $\mathrm{HPP}^{20}$, aunque es útil para evaluar la respuesta al tratamiento en ambos grupos ${ }^{42}$.

Los pacientes pueden tener hipoxemia leve, hipocapnia y un aumento del gradiente alveoloarterial de oxígeno ${ }^{15}$. La combinación de una presión parcial arterial de bióxido de carbono $<30 \mathrm{~mm} \mathrm{Hg}$ y un gradiente alveoloarterial de oxígeno $>20 \mathrm{~mm} \mathrm{Hg}$ tiene un valor predictivo negativo del $90 \%$ para el diagnóstico de HPP a una altitud de $100 \mathrm{~m}$ sobre el nivel del $\operatorname{mar}^{43,44}$. Sin embargo, no existe correlación entre los valores gasométricos y los parámetros hemodinámicos en esta población ${ }^{44}$. Las pruebas de funcionamiento respiratorio muestran un patrón restrictivo leve, con una disminución en la difusión de monóxido de carbono ${ }^{39}$. El electrocardiograma no se puede utilizar para diferenciar la HPP de otras formas de $\mathrm{HP}^{45}$, y la radiografía de tórax es normal en etapas tempranas, pero conforme progresa la enfermedad hay prominencia del tronco de la arteria pulmonar y de los hilios de manera bilateral, con una terminación abrupta de los vasos en la periferia y crecimiento del ventrículo derecho $(V D)^{46}$. Los datos en la tomografía de tórax incluso contrastada son similares a los distintos subtipos de HAP (datos de sobrecarga de presión de las cavidades derechas, patrón de perfusión en mosaico, un diámetro del tronco de la arteria pulmonar $>29 \mathrm{~mm}$, una relación $>1$ del diámetro de las arterias segmentarias y el bronquio correspondiente, y/o una relación $>1$ del diámetro del tronco de la arteria pulmonar y la aorta ascendente, especialmente en pacientes menores de 50 años) ${ }^{46}$. La gammagrafía ventilatoria/perfusoria pulmonar es normal o con defectos perfusorios difusos en parches $^{47}$, sin que se identifiquen defectos segmentarios ${ }^{48}$. La prueba de caminata de 6 minutos sirve para evaluar la CF de los pacientes con $\mathrm{HPP}^{49}$, y la distancia recorrida se relaciona con la CF y no con el índice de Child-Pugh ${ }^{4}$. Existe preocupación sobre la limitación de su utilidad en pacientes con ascitis y edema de tejidos blandos en las extremidades inferiores ${ }^{15}$, así como su falta de correlación con la SV de los pacientes ${ }^{4}$.

El ECOTT está indicado en los pacientes con HPo y síntomas sugestivos de HP, y en aquellos casos con HPo y $\mathrm{CH}$ evaluados para $\mathrm{THO}^{50}$. Aunque la mayoría de la literatura se enfoca en la medición de la PSVD y la PSAP, existen algoritmos de evaluación ecocardiográfica en pacientes con sospecha de HP, y en los que se resaltan otros parámetros de utilidad para el corazón derecho: velocidad telediastólica de regurgitación de la pulmonar, la relación del VD/ventrículo izquierdo, tiempo de aceleración del tracto de salida del VD, el desplazamiento del plano anular tricuspídeo en sístole, el tiempo de relajación isovolumétrica del VD, y el diámetro de la vena cava inferior con el grado de colapso inspiratorio. En caso de hallazgos anormales se deberán evaluar, además, el volumen de la aurícula derecha, índice de desempeño 
del VD, índices tisulares del VD, índice de excentricidad, gasto cardiaco, resistencia vascular pulmonar (RVP), datos de disfunción diastólica del ventrículo izquierdo, morfología/función valvular, y búsqueda de defectos cardiacos ${ }^{51}$.

La PSVD se calcula utilizando la velocidad pico de regurgitación tricuspídea $\left(\mathrm{V}_{\mathrm{TR}}\right)$ junto con la estimación de la presión de la aurícula derecha a través del grado de colapso inspiratorio de la vena cava inferior: $P S V D=4\left(V_{T R}\right)^{2}+$ presión de la aurícula derecha. Será importante descartar la presencia de un gradiente a través del tracto de salida del VD o de la válvula pulmonar (obstrucción) para poder considerar que la PSVD es igual a la PSAP ${ }^{52,53}$. Los resultados deben interpretarse con cuidado, pues hasta un $20 \%$ de los pacientes candidatos a THO tienen aumentos moderados de la presión pulmonar no debida a HPP. Además, a pesar de que existen datos que señalan que el ECOTT tiene una sensibilidad del $97 \%$ y una especificidad del $77 \%$ para detectar una $\mathrm{PMAP} \geq 35 \mathrm{~mm} \mathrm{Hg}$ por cateterismo cardiaco derecho (CTTD) en $\mathrm{HPP}^{53}$, también es importante señalar que, en un estudio, donde el $89 \%$ de los sujetos tenían HP por cateterismo cardiaco, hasta en el $50 \%$ de los casos la PSAP obtenida invasivamente tenía una diferencia $\geq 10 \mathrm{~mm} \mathrm{Hg}$ con respecto al ECOTT, y el problema radicaba, primordialmente, en la presión de la aurícula derecha estimada ${ }^{51,54}$.

Con lo mencionado previamente, no está bien definido el punto de corte de PSVD/PSAP en el ECOTT para recomendar la evaluación hemodinámica de los pacientes candidatos a THO. En un estudio se concluyó que una PSAP $>38 \mathrm{~mm} \mathrm{Hg}$ detectada por ECOTT es bastante sensible, específica y precisa, e incluso su especificidad se incrementa un poco si al valor de la PSAP se suma la dilatación del VD (diámetro telediastólico $\geq 3.3 \mathrm{~cm})^{55}$.

Actualmente, se recomienda clasificar por los hallazgos ecocardiográficos a los pacientes con sospecha de HP en: 1) baja probabilidad para HP ( $\mathrm{V}_{T R} \leq 2.8 \mathrm{~cm} / \mathrm{s}$ o no medible sin otros datos de HP); 2) probabilidad intermedia para HP (un $V_{T R} \leq 2.8 \mathrm{~cm} / \mathrm{s}$ o no medible con otros datos de $H P$, o un $V_{T R}$ de $2.9-3.4 \mathrm{~cm} / \mathrm{s}$ en el ECOTT), o 3) probabilidad alta para HP (un $V_{T R}$ de $2.9-3.4 \mathrm{~cm} / \mathrm{s}$ con otros datos de HP o un $V_{T R}>3.4 \mathrm{~cm} / \mathrm{s}$ ). Un paciente con HPo y sospecha de HP en el primer grupo solo requiere seguimiento ecocardiográfico (॥a/C), pues no se puede descartar el desarrollo de HPP en períodos cortos de 2 a 3 meses $^{13,28,55}$. En el segundo grupo se puede considerar el CTTD (॥a/B), mientras que en el tercero se debe someter el paciente a CTTD $(I / C)^{13}$. Por otro lado, están los pacientes con HPo evaluados para THO, y en los cuales no hay un corte bien definido de PSVD/PSAP para referir al paciente a CTTD. Existen algunos grupos que hacen sugerencias al respecto: 1) todos los pacientes candidatos a THO con una PSAP $\geq 45 \mathrm{~mm} \mathrm{Hg}$ (Asociación Americana para el Estudio de las Enfermedades Hepáticas o AASLD) ${ }^{56}$; 2) el algoritmo de la Clínica Mayo en Rochester, Minnesota, EUA utiliza una PSVD > $50 \mathrm{~mm} \mathrm{Hg}^{27}$; y 3) se pudiera considerar en aquellos casos con PSVD de $30-50 \mathrm{~mm} \mathrm{Hg}$ con dilatación y/o disfunción del VD por ECOTT ${ }^{57}$. La Asociación Europea para el Estudio del Hígado (EASL) no hace ninguna recomendación al respecto ${ }^{58}$.

Las mediciones en el CTTD permiten identificar a los pacientes del grupo 2 de HP y a aquellos con estado hiperdinámico ${ }^{45}$. El diagnóstico hemodinámico de la HPP es similar al de la HAP, es decir HP precapilar (tabla 2), pero con algunas particularidades (tabla 3 ). Aunque no se requiere para la definición actual de HAP, se recomienda conocer el valor de RVP. El valor normal es hasta 2 unidades Wood (UW), pero emplear un corte de $3 \mathrm{UW}$ o $240 \mathrm{dyn} / \mathrm{s} / \mathrm{cm}^{-5}$ se justifica por el hecho de que la probabilidad de HAP con RVP $<3$ UW es baja ${ }^{59}$. Para el caso de la HPP es un requisito que la RVP sea > 3 UW (> $240 \mathrm{dyn} / \mathrm{s} / \mathrm{cm}^{-5}$ ) para el diagnóstico ${ }^{45}$.

Los pacientes con HPo con PMAP $\geq 25 \mathrm{~mm} \mathrm{Hg}$ y presión de oclusión de la arteria pulmonar (POAP) o presión en cuña de la arteria pulmonar (PCAP) > $15 \mathrm{~mm} \mathrm{Hg}$, cumplen con la definición hemodinámica de HP poscapilar, sin embargo, la presencia de RVP > 3 UW orientan a la coexistencia de una HP precapilar ${ }^{13}$. En la serie de la Clínica Mayo, este grupo correspondió al $24 \%$ de los pacientes con $\mathrm{RVP}>3 \mathrm{UW}$. Lo más importante es que el $100 \%$ de estos casos tuvieron un gradiente transpulmonar de presión (GTP) $(G T P=P M A P-P O A P / P C A P)>12 \mathrm{~mm} \mathrm{Hg}$ (vs. $27 \%$ en los que tuvieron una RVP $<3 U W$ ). Otro dato relevante es que este subgrupo de HPP no tiene mayor grado de disfunción diastólica del ventrículo izquierdo, ni dilatación de la aurícula izquierda por el ECOTT ${ }^{29}$, lo que los diferenciaría de aquellos pacientes del grupo 2 de la OMS para HP.

Un dato interesante es que el GTP se ve influenciado por el flujo sanguíneo, la resistencia al flujo y las presiones de llenado de las cavidades izquierdas (determinantes de la PMAP), mientras que el gradiente diastólico de presión (GDP) (GDP=presión diastólica de la arteria pulmonar - POAP/PCAP) no está influenciado por los determinantes de la PMAP, ni de la POAP/PCAP, lo que parece ser una desventaja del GTP. Y aunque no se incluyeron pacientes con HPP cabe señalar que durante un estudio se encontró que en aquellos casos con HP poscapilar, un GTP > $12 \mathrm{~mm} \mathrm{Hg}$ y un GDP $\geq 7 \mathrm{~mm} \mathrm{Hg}$, la SV fue similar a la HAPI ${ }^{60}$. Hasta la fecha se recomienda el uso del GTP para el diagnóstico de HPP en el escenario de HP poscapilar.

La prueba de vasodilatación pulmonar aguda durante el CTTD (óxido nítrico inhalado, adenosina iv y epoprostenol iv), solo se recomienda en los pacientes con HAPI, HAPH y HAP inducida por fármacos/toxinas ${ }^{13}$. En la HPP hay un 0.7-1.5\% de respondedores a la prueba de vasodilatación pulmonar aguda (disminución $\geq 10 \mathrm{~mm} \mathrm{Hg}$ de la PMAP con un valor final de la misma de $\leq 40 \mathrm{~mm} \mathrm{Hg})^{17,61}$, aunque la tasa de respuesta persistente a los calcioantagonistas orales es del $0 \%{ }^{61}$. Además, los calcioantagonistas orales producen un incremento del GPVH debido a un aumento en el gasto cardiaco, lo que limita su uso de manera crónica ${ }^{62-64}$. Durante un estudio el uso de 5-mononitrato de isosorbide oral durante la prueba de vasodilatación pulmonar aguda produjo mejor respuesta vasodilatadora que el óxido nítrico inhalado y el epoprostenol iv ${ }^{65}$, aunque su uso no es regular.

No se recomienda la medición de rutina del GPVH $(G P V H=$ presión venosa hepática libre - presión venosa hepática en cuña) durante el CTTD, pero es recomendable para confirmar el diagnóstico de HPo. Se debe de medir la presión venosa hepática libre en el interior de una vena suprahepática, $2-4 \mathrm{~cm}$ de la desembocadura de la misma en la vena cava inferior, siendo importante que la diferencia de presión no sea mayor a $2 \mathrm{~mm} \mathrm{Hg}$ con respecto a la vena cava inferior. Posteriormente, se infla el globo del catéter, se corrobora la oclusión total mediante la inyección de $5 \mathrm{ml}$ de contraste y se espera 60 o más segundos para obtener la presión venosa hepática en cuña ${ }^{48,66}$. En pacientes con $\mathrm{CH}$ descompensada 
con ascitis refractaria, el GPVH en los casos con HPP fue de $35.2 \pm 2.5 \mathrm{~mm} \mathrm{Hg}$ (presión portal por punción $34.7 \pm 1.8 \mathrm{~mm}$ $\mathrm{Hg})^{26}$, mientras que en otro estudio los pacientes con HPo que fueron sometidos a biopsia hepática transyugular con una $P M A P \geq 25 \mathrm{~mm} \mathrm{Hg}, P O A P \leq 13 \mathrm{~mm} \mathrm{Hg}$ y $R V P>3 U W$ (15 sujetos) tuvieron un mediana del GPVH de 15(10) $\mathrm{mm}$ $\mathrm{Hg}^{25}$.

Es importante tener en cuenta que las condiciones hemodinámicas pulmonares pueden cambiar con el tiempo en los casos con HPP. Así, es bastante recomendable la evaluación con CTTD cada 12 meses en los candidatos a THO con HPP ${ }^{27}$. Aunque esto dependerá de las políticas de cada uno de los centros.

\section{Los diferentes pasos en el tratamiento}

Al igual que el resto de los pacientes con HAP el manejo consiste en medidas generales, terapia de soporte y terapia específica.

\section{Medidas generales}

Se debe evitar el embarazo. Se recomienda la inmunización anual para influenza y contra neumococo conforme a los lineamientos nacionales. Es importante el soporte psicosocial por el impacto que la enfermedad tiene sobre la calidad de vida de los pacientes. Además, se sugiere el uso de oxígeno en los vuelos en avión en pacientes en CF III-IV de la OMS y con una presión parcial de oxígeno $<60 \mathrm{~mm} \mathrm{Hg}$. En caso de ser necesaria una cirugía preferentemente deberá de emplearse anestesia epidural (difícil en el contexto de trombocitopenia). Y se debe de evitar la actividad física excesiva porque puede producir síntomas de bajo gasto cardiaco $^{13}$.

\section{Terapia de soporte}

El registro COMPERA, incluyó a 60 pacientes con HPP (el $30 \%$ anticoagulados), y no mostró que la anticoagulación oral brindara un beneficio en cuanto a la SV a 3 años en HAP no idiopática ${ }^{67}$.

Los pacientes con HPP e HPo hepática pueden presentar edema de tejidos blandos, así como ascitis. El manejo habitual en la HPo hepática es la combinación de furosemida con espironolactona en una relación de 40 y $100 \mathrm{mg}$, respectivamente (máximo $160 \mathrm{mg} / 400 \mathrm{mg})^{68}$. Así, aunque no existen estudios aleatorizados en pacientes con HAP, el uso de furosemida mejora la sintomatología y previene la descompensación de la falla cardiaca ${ }^{13}$. Además, existe evidencia del papel que la aldosterona puede desempeñar en la fisiopatología de la HAP, lo cual puede ser el sustento de que la combinación de espironolactona con ambrisentan, un antagonista del receptor de endotelina (ARE), tiene mejores resultados que el ARE aislado ${ }^{69}$. Es importante resaltar que en el contexto de la HPP el uso de diuréticos puede inducir encefalopatía hepática, lesión renal aguda, ginecomastia, desórdenes de electrólitos y calambres, principalmente, en las primeras semanas de uso. La frecuencia de estas complicaciones se puede disminuir utilizando como metas la disminución de $0,5 \mathrm{~kg}$ de peso al día si solo hay ascitis clínicamente demostrable, y hasta $1 \mathrm{~kg}$ si hay ascitis con edema periférico, disminuyendo la dosis una vez que no haya evidencia clínica de ascitis $^{70}$.

En este escenario, el uso de bloqueadores de canales de calcio no ha demostrado respuesta y se debe recordar que pueden producir incremento en la retención de líquidos, pueden reducir la función ventricular derecha e incrementar la presión portal por lo que se deben evitar en HPP ${ }^{71}$.

El oxígeno suplementario de manera ambulatoria solo debe prescribirse si la presión parcial de oxígeno $<60 \mathrm{~mm}$ $\mathrm{Hg}$ con evidencia de mejoría sintomática y de la oxigenación con su uso ${ }^{13}$.

Hay 2 fármacos cardiovasculares de interés: 1) la digoxina de la cual no se sabe su papel con el uso crónico en los pacientes con $\mathrm{HAP}^{13}$, y 2) los betabloqueadores, que se utilizan para la profilaxis del sangrado variceal, sin embargo, se ha demostrado que en casos con HPP moderada a grave su uso se asocia con una disminución de la distancia y mayor grado de disnea en la prueba de caminata de 6 minutos, y caída del gasto cardiaco en el CTTD ${ }^{72}$. Se deberá considerar evitar los betabloqueadores si es totalmente posible ${ }^{73}$ $y$, preferentemente, utilizar terapias endoscópicas si está indicado ${ }^{71,74}$.

La deficiencia de hierro es frecuente en la HAP, aunque no hay estudios controlados de los efectos de la terapia de sustitución $^{13}$. En los casos con HPo existe el riesgo inherente de pérdidas crónicas de sangre a través del tubo digestivo ${ }^{75}$, y aunque es recomendable la sustitución con hierro no se sabe los efectos a largo plazo en el hígado, ni la meta de hemoglobina en este contexto.

\section{Terapia específica}

En este apartado quedan incluidos los inhibidores de la fosfodiesterasa 5, los análogos de prostaciclina o prostanoides intravenosos, subcutáneos, inhalados u orales, los ARE, y los estimulantes de la guanilato ciclasa soluble.

Los pacientes con HPP han sido excluidos de casi todos los estudios aleatorizados y controlados, excepto del estudio PATENT (riociguat), que incluyó a 13 pacientes ( 2 con placebo y 11 con riociguat $2.5 \mathrm{mg} 3$ veces al día), aunque no se hizo ningún análisis de los subgrupos ${ }^{13,45,76}$. A pesar de esto existen múltiples estudios no aleatorizados que incluyen alrededor de 400 pacientes con HPP, y que muestran que la SV a 5 años de aquellos que recibieron alguna terapia específica fue del $40-68 \%$, mientras que sin esta solo fue del $2-14 \%{ }^{77}$.

El registro REVEAL mostró diferencias en cuanto al uso de terapia específica en la HPP. Así, los casos diagnosticados previamente recibían vasodilatadores específicos más frecuentemente que los de diagnóstico reciente (84 vs. $57 \%)$. A pesar de esto, la frecuencia de administración de estos fármacos en los casos previamente diagnosticados con HAPI/HAPH fue mayor que en sus contrapartes con HPP, lo que no se observó en los casos de reciente diagnóstico de ambos grupos de HAP. Por último, el grupo de terapia específica más utilizado en la HPP a los 3 y 12 meses de inclusión en el registro fue el de los inhibidores de la fosfodiesterasa 5 , seguidos por los análogos de prostaciclina intravenosos/subcutáneos, los análogos de prostaciclina inhalados/orales y el grupo menos empleado fue el de los $A R E^{20}$. La limitación en el uso de los ARE 
radica, principalmente, en el riesgo de hepatotoxicidad, sin embargo, la evidencia muestra que el perfil de seguridad en la HPP con $\mathrm{CH}$ es igual al de los pacientes con HAPI $\sin \mathrm{CH}$. La única recomendación es el seguimiento mensual de las pruebas de funcionamiento hepático ${ }^{45}$.

Con base en la historia natural y lo expuesto en los párrafos anteriores existe un consenso para utilizar la terapia específica en los pacientes con HPP. En cuanto a esto se pueden identificar 2 escenarios, el primero en el contexto de los pacientes candidatos a THO con HPP moderada a grave ${ }^{27}$, y el segundo en aquellos casos no candidatos a THO, pero que se encuentran por lo menos en CF II de la OMS ${ }^{13}$.

En la tabla 4 se exponen los diferentes vasodilatadores pulmonares de los que se tiene conocimiento de su uso en casos con HPP $13,42,74,76,78-86$.

Es interesante que la respuesta a la terapia específica, aparentemente, pudiera verse comprometida por la presencia de cortocircuitos portosistémicos espontáneos grandes ( $>10 \mathrm{~mm}$ ) en pacientes con $\mathrm{CH}$ (modelo para la enfermedad hepática avanzada $[M E L D] \pm 12$ ) y HPP moderada y grave. Su presencia es frecuente (71-87\%), y aunque no parecen estar relacionados con el desarrollo de HPP (presencia en sujetos con $\mathrm{CH}$ sin $\mathrm{HP}$ ), no es claro su papel hasta ahora ${ }^{87}$.

\section{El seguimiento}

En los pacientes con HAP en tratamiento con vasodilatadores específicos el seguimiento se ajustará a las características del sujeto y dependerá del centro, es decir que puede ser solo con revisiones clínicas, exámenes de laboratorios y ECOTT, o incluso con CTTD de manera regular $^{13}$. En el caso de los pacientes con HPP se recomienda la evaluación de la respuesta a la terapia específica cada 3 meses $^{57}$. Los resultados de la prueba de caminata de 6 minutos durante el seguimiento en este grupo pueden verse afectados por manifestaciones clínicas propias de ${\mathrm{la} \mathrm{CH}^{15}}^{15}$.

\section{El impacto del trasplante hepático}

A pesar de que el $90 \%$ de los casos de HPP en Occidente están relacionados con $\mathrm{CH}^{12,13}$, la indicación de $\mathrm{THO}$ dependerá de la gravedad de la enfermedad hepática/HPo (tabla 5) 27,56. A diferencia del síndrome hepatopulmonar, la HPP como una «indicación» de THO es controversial, pues la respuesta hemodinámica postrasplante no es predecible ${ }^{88}$. La mayoría de los pacientes con HPP pueden ser retirados de terapia vasodilatadora intravenosa luego del THO, y hasta la mitad pueden ser retirados de la terapia oral, sin embargo, no se sabe con claridad qué pacientes se pueden retirar exitosamente del tratamiento ${ }^{89}$. Además, hay que recordar que los candidatos a THO con HPP tienen 2.46 veces más riesgo de muerte que aquellos sin $\mathrm{HPP}^{83}$, y eso no es todo, ya que los casos que tienen una PMAP > $45 \mathrm{~mm} \mathrm{Hg}, 0>35 \mathrm{~mm}$ $\mathrm{Hg}$ y/o RVP $>400 \mathrm{dyn} / \mathrm{s} / \mathrm{cm}^{-5}$ (5UW) tienen una mortalidad perioperatoria del $50-100 \%{ }^{90}$.

La terapia específica desempeña un papel importante para el THO. Ashfaq et al. encontraron que los pacientes con HPP moderada a grave que recibieron terapia con vasodilatadores específicos (16 pacientes) tuvieron una SV del 75, 69 y $43 \%$ a uno, 2 y 5 años, respectivamente. Mientras que, de este grupo, aquellos que respondieron a la terapia y fueron trasplantados (11 casos) tuvieron una SV $>70 \%$ a partir del primer año de seguimiento ${ }^{84}$. Swanson et al. encontraron que en los pacientes con HPP tratados y sometidos a THO (9 casos) la SV fue $>70 \%$ a un año $y>65 \%$ hasta los 9 años de seguimiento ${ }^{91}$. Khaderi et al. en 7 pacientes con HPP tratados y con THO tuvieron una SV del $86 \%$ a 7.8 años $^{85}$.

Actualmente, se recomienda que los sujetos con HPP leve pueden recibir un THO sin necesidad de terapia específica, mientras que en el resto de los casos se ha propuesto utilizar la excepción del MELD de los EUA $27,71,83,89,90$.

1) HPP moderada a grave (diagnóstico hemodinámico):

a. $P M A P \geq 35 \mathrm{~mm} \mathrm{Hg}, R V P \geq 240 \mathrm{dyn} / \mathrm{s} / \mathrm{cm}^{-5}$ ( 3 UW), y $P O A P / P C A P \leq 15 \mathrm{~mm} \mathrm{Hg}$.

2) Mejoría documentada con la terapia específica vasomoduladora (mínimo 12 semanas):

a. $P M A P<35 \mathrm{~mm} \mathrm{Hg}, R V P<400 \mathrm{dyn} / \mathrm{s} / \mathrm{cm}^{-5}$ (5UW), y función satisfactoria del VD por ECOTT (definición no estandarizada que depende del centro).

3) Se deberá actualizar cada 3 meses la excepción de MELD si se cumplió el criterio hemodinámico de respuesta (punto 2).

a. Agregar 2 puntos del puntaje de MELD

La Red de Procuración y Trasplante de Órganos (EUA) recomienda que a estos pacientes se les asigne 22 puntos de MELD de manera inicial ${ }^{71,92}$ para acortar los tiempos de espera para un THO. A pesar de las recomendaciones, menos de la mitad de los pacientes con excepción de MELD y HPP en la lista de espera en los EUA cumplen estos criterios estandarizados ${ }^{83}$.

No se puede concluir nada acerca de la utilidad del trasplante combinado pulmón-hígado o pulmón-corazón-hígado en pacientes con HPP (tabla 5), pues hasta el 2014 se habían realizado 58 trasplantes de este tipo por diversas indicaciones (SV de 20 a $43 \%$ a 1 año) ${ }^{93,94}$, y hasta el 2011 solo 10 en HPP ( 6 trasplantes combinados pulmón-hígado y 4 trasplantes combinados pulmón-corazón-hígado) con 2 fallecimientos en las primeras $24 \mathrm{~h}$ (choque cardiogénico) y SV de 2-140 meses en el resto ${ }^{86}$.

En algunos casos existe una normalización hemodinámica postrasplante, sin embargo, se desconoce si realmente refleja la curación de la enfermedad vascular pulmonar ${ }^{88}$. A pesar del optimismo en algunos grupos, el porcentaje de pacientes que permanecen con terapia específica es variable (18-50\%), no se sabe la razón, pero pudiera estar relacionada con la etiología de la HPo (mejor respuesta en enfermedad hepática asociada a ingesta de alcohol) y/o a la definición de «mejoría» hemodinámica postrasplante ${ }^{85}$.

Finalmente, la HPP es un reto para el anestesiólogo, pues aumenta el riesgo de complicaciones trans- y postoperatorias en el THO. Los cambios mayores en la hemodinámica pulmonar con la repercusión se consideran un reto clínico ${ }^{71}$. Los momentos críticos son durante la inducción anestésica (disminución del tono vasomotor, presión positiva de la ventilación mecánica invasiva), la fase preanhepática (infusión de cristaloides, coloides y productos sanguíneos), la fase anhepática con el pinzamiento de la vena cava, vena porta y arteria hepática (caída del gasto cardiaco del 40-50\%), y la reperfusión del órgano (aumento en la PMAP asociado a sustancias vasoactivas e incremento del gasto cardiaco $)^{34,89}$. 


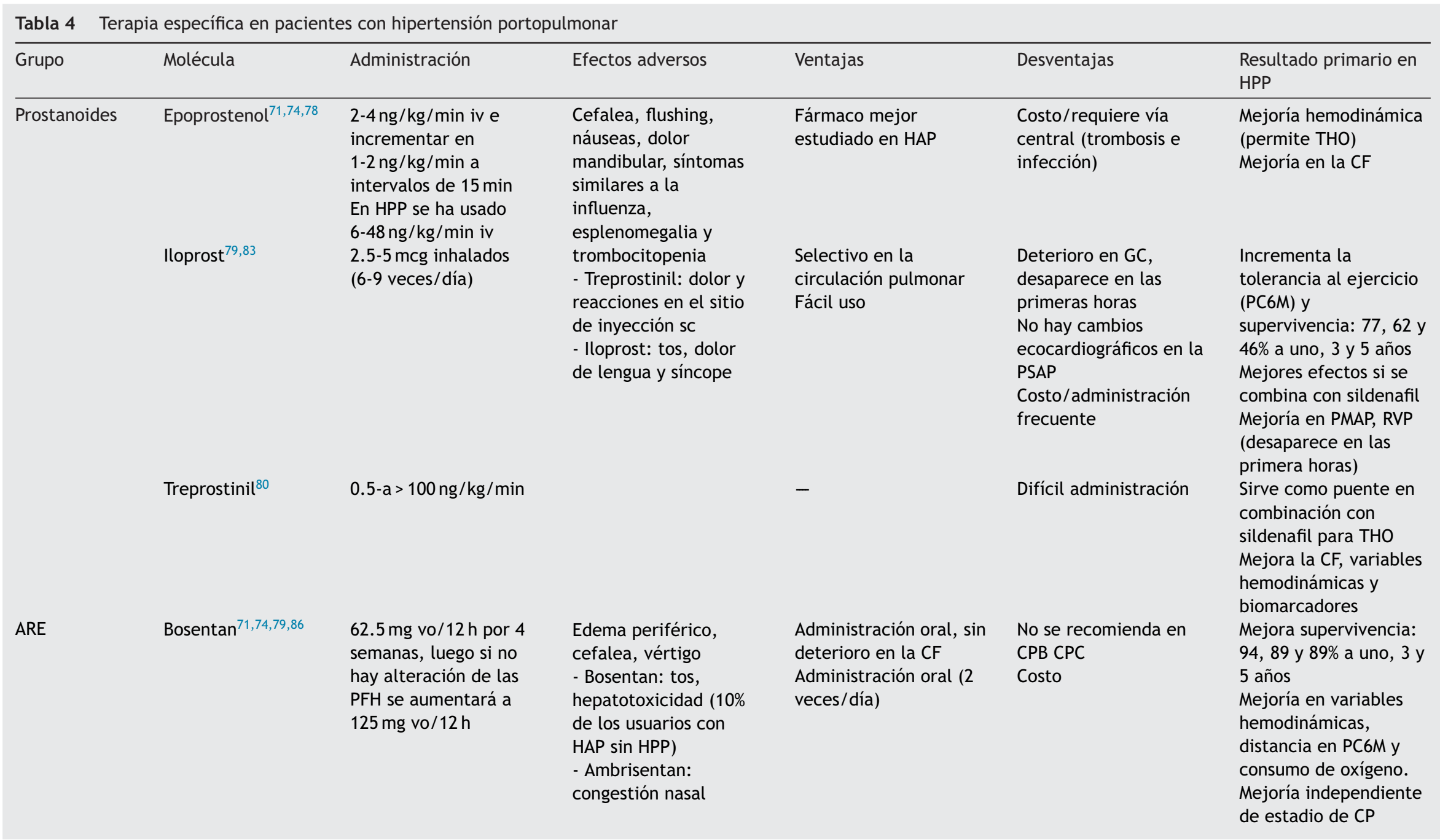




\begin{tabular}{|c|c|c|c|c|c|c|}
\hline Grupo & Molécula & Administración & Efectos adversos & Ventajas & Desventajas & $\begin{array}{l}\text { Resultado primario en } \\
\text { HPP }\end{array}$ \\
\hline & Ambrisentan $^{84}$ & $5-10 \mathrm{mg}$ vo $/ 24 \mathrm{~h}$ & & $\begin{array}{l}\text { Una sola dosis al día, } \\
\text { no altera los exámenes } \\
\text { de laboratorio }\end{array}$ & Costo & $\begin{array}{l}\text { En HPP moderada a } \\
\text { grave, mostró mejoría } \\
\text { en perfil hemodinámico } \\
\text { (PMAP, RVP y GC) y } \\
\text { niveles de BNP } \\
\text { Mejoría en clase } \\
\text { funcional }\end{array}$ \\
\hline EGC & Riociguat $^{13,76}$ & $0.5-2.5 \mathrm{mg}$ vo $/ 8 \mathrm{~h}$ & $\begin{array}{l}\text { Cefalea, dispepsia, } \\
\text { edema, náuseas, } \\
\text { mareo, diarrea, } \\
\text { vómito, hipotensión } \\
\text { arterial }\end{array}$ & Administración oral & $\begin{array}{l}\text { Costo. Tiempo de } \\
\text { escalamiento de la } \\
\text { dosis } \\
\text { No se ha estudiado } \\
\text { como puente para THO }\end{array}$ & $\begin{array}{l}\text { Mejoría en la CF, } \\
\text { distancia en la PC6M, } \\
\text { de algunas variables } \\
\text { hemodinámicas y } \\
\text { retraso en el deterioro } \\
\text { clínico }\end{array}$ \\
\hline
\end{tabular}

ARE: antagonista del receptor de endotelina; BNP: péptido natriurético cerebral; CF: clase funcional; CP: Child-Pugh; CPB: Child-Pugh B; CPC: Child-Pugh C; GC: gasto cardiaco; HAP: hipertensión arterial pulmonar; HPP: hipertensión portopulmonar; IFD-5: inhibidores de la fosfodiesterasa 5; iv: intravenoso; NT-proBNP: fragmento N terminal del péptido natriurético cerebral; PC6M: prueba de caminata de 6 minutos; PFH: pruebas de funcionamiento hepático; PMAP: presión media de arteria pulmonar; PSAP: presión sistólica de la arteria pulmonar; RVP: resistencia vascular pulmonar; sc: subcutánea; THO: trasplante hepático ortotópico; vo: vía oral. 
Tabla 5 Indicaciones para trasplante hepático ortotópico y combinado

\begin{tabular}{|c|c|}
\hline Hepático 27,79 & $\begin{array}{l}\text { Descompensación de la cirrosis hepática: ascitis, } \\
\text { encefalopatía hepática, sangrado variceal; o } \\
\text { Un puntaje del modelo para la enfermedad hepática } \\
\text { avanzada o MELD } \geq 15\end{array}$ \\
\hline \multicolumn{2}{|r|}{ 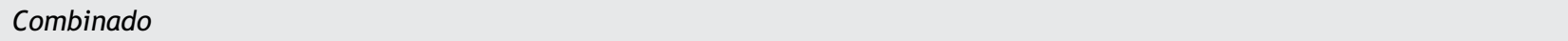 } \\
\hline Pulmón-hígado ${ }^{84}$ & $\begin{array}{l}\text { Enfermedad vascular pulmonar con: } \\
\text { a. CF III o IV de la New York Heart Association a pesar de } \\
\text { por lo menos } 3 \text { meses de terapia combinada incluidos } \\
\text { prostanoides } \\
\text { b. Índice cardiaco }<2 \mathrm{l} / \mathrm{min} / \mathrm{m}^{2} \\
\text { c. PAD }>15 \mathrm{~mm} \mathrm{Hg} \\
\text { d. }<350 \mathrm{~m} \text { (PC } 6 \mathrm{M}) \\
\text { e. Desarrollo de hemoptisis o derrame pericárdico } \\
\text { significativos, o datos de falla cardiaca derecha } \\
\text { progresiva (insuficiencia renal, hiperbilirrubinemia, } \\
\text { aumento del BNP o ascitis recurrente) } \\
\text { Con evidencia histológica de cirrosis más un } \\
\text { GPVH }>10 \mathrm{~mm} \text { Hg } \\
\text { a. Evitar si: albúmina }<2 \mathrm{~g} / \mathrm{dl} \text {, INR }>1.8 \text { o en presencia } \\
\text { de ascitis o encefalopatía grave }\end{array}$ \\
\hline Pulmón-corazón-hígado ${ }^{84}$ & $\begin{array}{l}\text { Arriba mencionado más: } \\
\text { a. Disfunción miocárdica o defectos, o defectos de las } \\
\text { cavidades cardiacas irreparables }\end{array}$ \\
\hline
\end{tabular}

BNP: péptido natriurético cerebral; CF: clase funcional; GPVH: gradiente de presión venosa hepática; INR: cociente normalizado internacional; MELD: modelo para la enfermedad hepática avanzada; PAD: presión de la aurícula derecha; PC6M: prueba de caminata de 6 minutos.

Así, junto con una cirugía $>12 \mathrm{~h}$, la presencia de una presión arterial media $<40 \mathrm{~mm} \mathrm{Hg}$ (un episodio de $\geq 2$ mins) y una PMAP $>40 \mathrm{~mm} \mathrm{Hg}$ ( 3 episodios de $\geq 2$ min separados entre sí) durante el transoperatorio, se asocian con pobre funcionamiento del injerto tempranamente, disfunción primaria del injerto o muerte por causas hemodinámicas (infarto del miocardio o arritmias cardiacas $)^{95}$. En ocasiones es necesario que se empleen algunos fármacos cardiovasculares como inotrópicos, vasopresores e incluso un sistema de circulación extracorpórea venovenoso ${ }^{34,89}$. De manera inicial en la fase postoperatoria se debe ser restrictivo con el manejo de líquidos intravenosos en este grupo de pacientes, y en caso de haber deterioro de la función renal se debe considerar la hemodiálisis venovenosa continua ${ }^{96}$. Además, hay que recordar que la respuesta hemodinámica pulmonar postrasplante es impredecible, así que se debe de tener en consideración la posibilidad de utilizar terapias específicas. Aunque no existen estudios aleatorizados para el manejo de la HP en el postoperatorio, junto con las medidas previamente mencionadas se puede emplear para el control de la PMAP y RVP prostanoides (epoprostenol iv e iloprost inhalado), óxido nítrico inhalado (costoso, con pobre respuesta en los pacientes con HP «fija», riesgo de síndrome de supresión -hipotensión arterial y deterioro de la oxigenación-), y sildenafil (respuesta equiparable al epoprostenol iv). Asimismo se ha planteado la posibilidad de utilizar estos fármacos en combinación en búsqueda de un efecto aditivo (epoprostenol iv +óxido nítrico inhalado, iloprost inhalado + óxido nítrico inhalado, sildenafil + óxido nítrico inhalado para evitar el síndrome de supresión). Por último, se deberá de mantener medidas de ventilación mecánica invasiva con metas de protección pulmonar y optimizar la oxigenación ${ }^{97}$.

\section{Conclusiones}

La frecuencia de la HPP en México se desconoce, y su estudio se encuentra en una etapa inicial. Probablemente, en cuanto el número de THO aumente en nuestro país se tendrán cifras aproximadas de la prevalencia de este padecimiento. El diagnóstico, finalmente, debe de ser hemodinámico, y durante la valoración diagnóstica en el escenario de sospecha de HP, así como en los candidatos a THO que cumplan con el criterio ecocardiográfico utilizado por el centro hospitalario para la evaluación hemodinámica. El uso de las terapias específicas parece tener un papel en el manejo de cierto grupo de pacientes candidatos a THO con HPP, así como en el tratamiento trans- y postoperatorio. En México existen algunos centros hospitalarios con unidades especializadas en el diagnóstico y tratamiento de la enfermedad vascular pulmonar. Habrá que crear un enlace entre estas y los centros con alto volumen de pacientes con $\mathrm{CH}$, además con los equipos de THO que en el país existan, teniendo en cuenta que no es necesario que cada centro de THO cuente con una unidad de enfermedad vascular pulmonar, sino que se haga efectiva y ágil la mecánica de referencia.

\section{Financiación}

Ninguna.

\section{Conflicto de intereses}

Los autores declaran no tener ningún conflicto de intereses. 


\section{Bibliografía}

1. Montaño-Loza A, Meza-Junco J. Patogénesis de la hipertensión portal. Rev Invest Clin. 2005;57:596-607.

2. Narváez-Rivera RM, Cortez-Hernández CA, González-González JA, et al. Mexican consensus on portal hypertension. Rev Gastroenterol Mex. 2013;78:92-113.

3. Goel A, Madhu K, Zachariah U, et al. A study of etiology of portal hypertension in adults (including the elderly) at a tertiary centre in southern India. Indian J Med Res. 2013;137:922-7.

4. Le Pavec J, Souza R, Herve P, et al. Portopulmonary hypertension. Survival and prognostic factors. Am J Respir Crit Care Med. 2008;178:637-43.

5. Herve P, Le Pavec J, Sztrymf B, et al. Pulmonary vascular abnormalities in cirrhosis. Best Pract Res Clin Gastroenterol. 2007;21:141-59.

6. Ridaura-Sanz C, Mejía-Hernández C, López Corella E. Portopulmonary hypertension in children. A study in pediatric autopsies. Arch Med Res. 2009;40:635-9.

7. Mokdad AA, Lopez AD, Shahraz S, et al. Liver cirrhosis mortality in 187 countries between 1980 and 2010: A systematic analysis. BMC Medicine. 2014;12:145.

8. Patrones de mortalidad en España, 2011. Madrid: Ministerio de Sanidad, Servicios Sociales e Igualdad; 2014 [consultado 15 Feb 2016]. Disponible en: http://www.mssi.gob.es/estadEstudios/ estadisticas/estadisticas/estMinisterio/mortalidad/mortalidad. htm

9. www.cdc.gov/minorityhealth/populations/REMP/hispanic.htlm [consultado 15 Feb 2016].

10. Heron M. Deaths: Leading causes for 2010. Natl Vital Stat Rep. 2013;62:1-96.

11. Frontera de Estados Unidos y México. Salud en las Américas 2012 [consultado 15 Feb 2016]. Disponible en: http://www.mssi. gob.es/estadEstudios/estadisticas/estadisticas/estMinisterio/ mortalidad/mortalidad.htm

12. www.inegi.org.mx/ [sonsultado 16 Dic 2015].

13. Galiè N, Humbert M, Vachiery JL, et al. 2015 ESC/ERS Guidelines for the diagnosis and treatment of pulmonary hypertension: The Joint Task Force for the Diagnosis and Treatment of Pulmonary Hypertension of the European Society of Cardiology (ESC) and the European Respiratory Society (ERS): Endorsed by: Association for European Pediatric and Congenital Cardiology (AEPC), International Society for Heart and Lung Transplantation (ISHLT). Eur Heart J. 2016;37:67-119.

14. Vonk-Noordegraaf A, Haddad F, Chin KM, et al. Right heart adaptation to pulmonary arterial hypertension. J Am Coll Cardiol. 2013;62:D22-33.

15. Budhiraja R, Hassoun PM. Portopulmonary hypertension. A tale of two circulations. Chest. 2003;123:562-76.

16. Safdar Z, Bartolome S, Sussman N. Portopulmonary hypertension: An update. Liver Transpl. 2012;18:881-91.

17. Humbert M, Sitbon O, Chaouat A, et al. Pulmonary arterial hypertension in France. Results from a National Registry. Am J Respir Crit Care Med. 2006;173:1023-30.

18. Tueller C, Stricker H, Soccal P, et al. Epidemiology of pulmonary hypertension: New data from the Swiss registry. Swiss Med Wkly. 2008;138:379-84

19. Hoeper MM, Gibbs SR. The changing landscape of pulmonary arterial hypertension and implications for patient care. Eur Respir Rev. 2014;23:450-7

20. Krowka MJ, Miller DP, Barst RJ, et al. Portopulmonary hypertension: A report from the US-based REVEAL Registry. Chest. 2012;141:906-15.

21. Kawut SM, Krowka MJ, Trotter JF, et al. Clinical risk factors for portopulmonary hypertension. Hepatology. 2008;48:196-203.

22. McDonnell PJ, Toye PA, Hutchins GM. Primary pulmonary hypertension and cirrhosis: Are they related? Am Rev Respir Dis. 1983;127:437-41.
23. Porres-Aguilar M, Altamirano JT, Torre-Delgadillo A, et al. Portopulmonary hypertension and hepatopulmonary syndrome: A clinician-oriented overview. Eur Respir Rev. 2012;21:223-33.

24. Rodríguez-Roisin R, Krowka MJ, Fallon MB. Pulmonary-hepatic vascular disorders (PHD). Eur Respir J. 2006;24:861-80.

25. Hadengue A, Benhayoun MK, Lebrec D, et al. Pulmonary hypertension complicating portal hypertension: Prevalence and relation to splanchnic hemodynamics. Gastroenterology. 1991;100:520-8.

26. Benjaminov FS, Prentice M, Sniderman KW, et al. Portopulmonary hypertension in decompensated cirrhosis with refractory ascites. Gut. 2003;52:1355-62.

27. Krowka MJ, Rodriguez-Roisin R. Portopulmonary hypertension: A consequence of portal hypertension. En: Hoeper MM, Humbert M, editores. European Respiratory Monograp: Pulmonary hypertension. Reino Unido: European Respiratory Society; 2012. p. $58-70$.

28. Colle IO, Moreau R, Godinho E, et al. Diagnosis of portopulmonary hypertension in candidates for liver transplantation: A prospective study. Hepatology. 2003;37:401-9.

29. Krowka MJ, Swanson KI, Frantz RP, et al. Portopulmonary hypertension: Results from a 10-year screening algorithm. Hepatology. 2006;44:1502-10.

30. Dadfarmy S, Berkowitz R, Kim B, et al. Differentiating pulmonary arterial and pulmonary venous hypertension and the implications for therapy. Congest Heart Fail. 2010;16:287-91.

31. Machicao VI, Balakrishnan M, Fallon MB. Pulmonary complications in chronic liver disease. Hepatology. 2014;59:1627-37.

32. Huffmyer JL, Nemergut EC. Respiratory dysfunction and pulmonary disease in cirrhosis and other hepatic disorders. Respir Care. 2007;52:1030-6.

33. Wong F. Portopulmonary hypertension in cirrhosis: The pathogenetic challenge. Gut. 2005;54:309.

34. Aldenkortt F, Aldenkortt M, Caviezel L, et al. Portopulmonary hypertension and hepatopulmonary syndrome. World J Gastroenterol. 2014;20:8072-81.

35. Tuder RM, Cool CD, Geraci MW, et al. Prostacyclin synthase expression is decreased in lungs from patients with severe pulmonary hypertension. Am J Respir Crit Care Med. 1999;159:1925-32.

36. Pellicelli AM, Barbaro G, Puota C, et al. Plasma cytokines and portopulmonary hypertension in patients with cirrhosis waiting for orthotopic liver transplantation. Angiology. 2010;61: 802-6.

37. Roberts KE, Fallon MB, Krowka MJ, et al. Genetic risk factors for portopulmonary hypertension in patients with advanced liver disease. Am J Respir Crit Care Med. 2009;179:835-42.

38. Grewal P, Martin P. Pretransplant management of the cirrhotic patient. Clin Liver Dis. 2007;11:431-49.

39. Golbin JM, Krowka MJ. Portopulmonary hypertension. Clin Chest Med. 2007;28:203-18.

40. Fijalkowska A, Kurzyna M, Torbicki A, et al. Serum N-terminal brain natriuretic peptide as a prognostic parameter in patients with pulmonary hypertension. Chest. 2006;129:1313-21.

41. Souza R, Jardim C, Fernandes CJC, et al. NT-proBNP as a tool to stratify disease severity in pulmonary arterial hypertension. Respir Med. 2007;101:69-75.

42. Reichenberger F, Voswinckel R, Steveling E, et al. Sildenafil treatment for portopulmonary hypertension. Eur Respir J. 2006;28:563-7.

43. Kuo PC, Plotkin JS, Jonson LB, et al. Distinctive clinical features of portopulmonary hypertension. Chest. 2002;112:980-6.

44. Swanson KL, Krowka MJ. Arterial oxygentation associated with portopulmonary hypertension. Chest. 2002;121:1869-75.

45. Liberal R, Grant CR, Baptista R, et al. Porto-pulmonary hypertension: A comprehensive review. Clin Res Hepatol Gastroenterol. 2015;39:157-67. 
46. Kim YK, Kim Y, Shim SS. Thoracic complications of liver cirrhosis: Radiologic findings. Radiographics. 2009;29:825-37.

47. Khan AN, Al-Jahdali $\mathrm{H}$, Abdullah K, et al. Pulmonary vascular compllications of chronic liver disease: Pathophysiology, imaging, and treatment. Ann Thorac Med. 2011;6:57-65.

48. Porres-Aguilar M, Mukherjee D. Portopulmonary hypertension: An update. Respirology. 2015;20:235-42.

49. Saleemi S. Portopulmonary hypertension. Ann Thoracic Med. 2010;5:5-9.

50. Krowka MJ. Evolving dilemmas and management of portopulmonary hypertension. Semin Liver Dis. 2006;26:265-72.

51. Howard LS, Grapsa J, Dawson D, et al. Echocardiographic assessment of pulmonary hypertension: Standard operating procedure. Eur Respir Rev. 2012;21:239-48.

52. Rudski LG, Lai WW, Afilalo J, et al. Guidelines for the echocardiographic assessment of the right heart in adults: A report from the American Society of Echocardiography endorsed by the European Association of Echocardiography, a registered branch of the European Society of Cardiology, and the Canadian Society of Echocardiography. J Am Soc Echocardiogr. 2010;11:685-713.

53. Garg A, Armstrong WF. Echocardiography in liver transplant candidates. JACC Cardiovasc Imaging. 2013;6:105-19.

54. Fisher MR, Forfia PR, Chamera E, et al. Accuracy of doppler echocardiography in the hemodynamic assessment of pulmonary hypertension. Am J Respir Crit Care Med. 2009;179:615-21.

55. Raevens S, Colle I, Reyntjens K, et al. Echocardiography for the detection of portopulmonary hypertension in liver transplant candidates: An analysis of cutoff values. Liver Transpl. 2013;19:602-10.

56. Martin P, DiMartini A, Feng S, et al. Evaluation for liver transplantation in adults: 2013 practice guideline by the American Association for the Study of Liver Diseases and the American Society of Transplantation. Hepatology. 2014;59:1144-65.

57. Bozbas SS, Bozbas H. Portopulmonary hypertension in liver transplant candidates. World J Gastroenterol. 2016;22:2024-9.

58. European Association for the Study of the Liver. EASL clinical practice guidelines: Liver transplantation. J Hepatol. 2016;64:433-85.

59. Hoeper MM, Bogaard HJ, Condliffe R, et al. Definitions and diagnosis of pulmonary hypertension. J Am Coll Cardiol. 2013;62:D42-50.

60. Gerges C, Gerges M, Lang MB, et al. Diastolic pulmonary vascular pressure gradient: A predictor of prognosis in out-of-proportion pulmonary hypertension. Chest. 2013;143:758-66.

61. Tonelli AR, Alnuaimat H, Mubarak K. Pulmonary vasodilator testing and use of calcium channel blockers in pulmonary arterial hypertension. Respir Med. 2010;104:481-96.

62. Hernández-Guerra M, García-Pagán JC, Bosch J. Increased hepatic resistance. A new target in the pharmacologic therapy of portal hypertension. J Clin Gastroenterol. 2005;39:S131-7.

63. Navasa M, Bosch J, Reichen J, et al. Effects of verapamil on hepatic and systemic hemodynamics and liver function in patients with cirrhosis and portal hypertension. Hepatology. $1988 ; 8: 850-4$.

64. Koshy A, Hadengue A, Lee SS, et al. Possible deleterious hemodynamic effect of nifedipine on portal hypertension in patients with cirrhosis. Clin Pharmacol Ther. 1987;42:295-8.

65. Ding SH, Liu J, Wang JP. Efficacy of beta-adrenergic blocker plus 5-isosorbide mononitrate and endoscopic band ligation for prophylaxis of esophageal variceal rebleeding: A meta-analysis. World J Gastroenterol. 2009;15:2151-5.

66. Berzigotti A, Seijo S, Reverter E, et al. Assessing portal hypertension in liver diseases. Expert Rev Gastroenterol Hepatol. 2013;7:141-55.

67. Olsson KM, Delcroix M, Ghofrani A, et al. Anticoagulation and survival in pulmonary arterial hypertension: Results from the Comparative, Prospective Registry of Newly Initiated
Therapies for Pulmonary Hypertension (COMPERA). Circulation. 2014;129:57-65.

68. Runyon BA. Management of adult patients with ascites due to cirrosis: Update 2012. Hepatology. 2013 [consultado 1 May 2016]. Disponible en: http://www.aasld.org/sites/ default/files/guideline_documents/AASLDPracticeGuideline AsciteDuetoCirrhosisUpdate2012Edition4_.pdf

69. Maron BA, Waxman AB, Opotowsky AR, et al. Effectiveness of spironolactone plus ambrisentan for treatment of pulmonary arterial hypertension (from the [ARIES] study 1 and 2 trials). Am J Cardiol. 2013;112:720-5.

70. Lenz K, Buder R, Kapun L, et al. Treatment and management of ascites and hepatorenal syndrome: An update. Ther Adv Gastroenterol. 2015;8:83-100.

71. Krowka MJ, Fallon MB, Kawut SM, et al. International Liver Transplant Society Practice Guidelines: Diagnosis and management of hepatopulmonary syndrome and portopulmonary hypertension. Transplantation. 2016;100:1440-52.

72. Provencher S, Herve P, Jais X, et al. Deleteroius effects of B-blockers on exercise capacity and hemodynamics in patients with portopulmonary hypertension. Gastroenterology. 2006;130:120-6.

73. Swanson KL, Krowka MJ. Screen for portopulmonary hypertension, especially in liver transplant candidates. Cleve Clin J Med. 2008;75:121-36.

74. Savale L, O'Callaghan DS, Magnier R, et al. Current management approaches to portopulmonary hypertension. Int J Clin Pract. 2011;65 Suppl 169:11-8.

75. Gisbert JP, Gomollón F. Spectrum of anemia associated with chronic liver disease. World J Gastroenterol. 2009;15:4653-8.

76. Ghofrani HA, Galiè N, Grimminger F, et al. Riociguat for the treatment of pulmonary arterial hypertension. N Engl J Med. 2013;369:330-40.

77. Krowka MJ, Cartin-Ceba R. Portopulmonary hypertension: Formidable dual threat versus hopeful dual therapy. Liver Transpl. 2014;20:635-6.

78. Krowka MJ, Frantz RP, McGoon MD, et al. Improvement in pulmonary hemodynamics during intravenous epoprostenol (prostacyclin): A study of 15 patients with moderate to severe portopulmonary hypertension. Hepatology. 1999;30:641-8.

79. Krowka MJ, Fallon MB, Mulligan DC, et al. Model for End-Stage Liver Disease (MELD) exception for portopulmonary hypertension. Liver Transpl. 2006;12 12 Suppl 3:S114-6.

80. Saggar R, Waxman AB, Bartolome S, et al. An open-label study to assess the safety and efficacy of treprostinil to facilitate liver transplantation in patients with portopulmonary hypertension (POPH). Am J Respir Crit Care Med. 2014;189:A4797.

81. Hemnes AR, Robbins IM. Sildenafil monotherapy in portopulmonary hypertension can facilitate liver transplantation. Liver Transp. 2009;1:5-19.

82. Gough MS, White RJ. Sildenafil therapy is associated with improved hemodynamics in liver transplantation candidates with pulmonary arterial hypertension. Liver Transpl. 2009;15:30-6.

83. Porres-Aguilar M, Bartolome SD, Duarte-Rojo A. MELD exception for liver transplantation in portopulmonary hypertension: Current implementation and future considerations. Ann Hepatol. 2014;13:719-21.

84. Ashfaq M, Chinnakotla S, Rogers L, et al. The impact of treatment of portopulmonary hypertension on survival following liver transplantation. Am J Transplant. 2007;7:1258-64.

85. Khaderi S, Khan R, Safdar Z, et al. Long-term follow-up of portopulmonary hypertension patients after liver transplantation. Liver Transpl. 2014;20:724-7.

86. Scouras NE, Matsusaki T, Boucek CD, et al. Portopulmonary hypertension as an indication for combined heart, lung, and liver or lung and liver transplantation: Literature review and case presentation. Liver Transpl. 2011;17:137-43. 
87. Talwalkar JA, Swanson KL, Krowka MJ, et al. Prevalence of spontaneous portosystemic shunts in patients with portopulmonary hypertension and effect on treatment. Gastroenterology. 2011;141:1673-9.

88. Krowka MJ, Wiesner RH, Heimbach JK. Pulmonary contraindications, indications and MELD exceptions for liver transplantation: A contemporary view and look forward. J Hepatol. 2013;59:367-74.

89. Fussner LA, Krowka MJ. Current approach to the diagnosis and management of portopulmonary hypertension. Curr Gastroenterol Rep. 2016;18:29.

90. Goldberg DS, Batra S, Sahay S, et al. MELD exceptions for portopulmonary hypertension: Current policy and future implications. Am J Transplant. 2014;14:2081-7.

91. Swanson KL, Wiesner RH, Nyberg SL, et al. Survival in portopulmonary hypertension: Mayo Clinic experience categorized by treatment subgroups. Am J Transplant. 2008;8:2445-53.

92. Runo JR. Liver transplantation for portopulmonary hypertension. Clin Liver Dis. 2014;4:74-6.
93. Yi SG, Burroughs SG, Loebe M, et al. Combined lung and liver transplantation: Analysis of a single-centre experience. Liver Transpl. 2014;20:46-53.

94. Weill D, Benden C, Corris PA, et al. A consensus document for the selection of lung transplant candidates: 2014-An update from the Pulmonary Transplantation Council of the International Society for Heart and Lung Transplantation. J Heart Lung Transplant. 2015;34:1-15.

95. Reich DL, Wood RK, Emre S, et al. Association of intraoperative hypotension and pulmonary hypertension with adverse outcomes after orthotopic liver transplantation. J Cardiothorac Vasc Anesth. 2003;17:699-702.

96. Steadman RH, Ramsay AE. (2015). Portopulmonary hypertension and hepatopulmonary syndrome. En: Busuttil RW, Klintmalm GB, editores. Transplantation of the liver. 2015 [consultado $14 \mathrm{Abr}$ 2016]. Disponible en: http: / /books.google.com

97. Hill NS, Roberts KR, Preston IR. Postoperative pulmonary hypertension: Atiology and treatment of a dangerous complication. Respir Care. 2009;54:958-68. 